\title{
WNT/ $\beta$-catenin pathway activation via Wnt1 overexpression and Axin1 downregulation correlates with cadherin-catenin complex disruption and increased lymph node involvement in micropapillary-predominant lung adenocarcinoma
}

\author{
Liang Zhu ${ }^{1,2,3}$, Shifeng Yang ${ }^{1,2,3}$, Linfeng Zheng ${ }^{1,2,3}$, Gu Zhang ${ }^{1,2,3}$, Guoping Cheng ${ }^{1,2,3}$ \\ ${ }^{1}$ Institute of Cancer and Basic Medicine (ICBM), Chinese Academy of Sciences, Hangzhou, China; ${ }^{2}$ Department of Pathology, Cancer Hospital of \\ the University of Chinese Academy of Sciences, Hangzhou, China; ${ }^{3}$ Department of Pathology, Zhejiang Cancer Hospital, Hangzhou, China \\ Contributions: (I) Conception and design: L Zhu, G Cheng; (II) Administrative support: G Zhang; (III) Provision of study materials or patients: S \\ Yang; (IV) Collection and assembly of data: S Yang, L Zheng; (V) Data analysis and interpretation: L Zhu, G Cheng; (VI) Manuscript writing: All \\ authors; (VII) Final approval of manuscript: All authors. \\ Correspondence to: Guoping Cheng. Department of Pathology, Cancer Hospital of the University of Chinese Academy of Sciences, Hangzhou 310022, \\ China. Email: chenggp@zjcc.org.cn.
}

\begin{abstract}
Background: Micropapillary-predominant adenocarcinoma (MPA) of the lung is associated with extensive lymph node involvement and rapid terminal metastasis. However, this subtype has been recognized for only a few years, and there have been few studies of the molecular mechanisms associated with its highly invasive behaviors.

Methods: The present study utilized immunohistochemical staining of surgically resected tissue blocks of MPA and lepidic-predominant lung adenocarcinoma to quantify the expression of specific biological markers in the WNT/ $\beta$-catenin pathway and evaluate their influence on the lymph nodes invasion of these two types of lung adenocarcinomas.

Results: Our findings revealed that disruption of the cell membrane cadherin-catenin complex, which weakens the tumor cell adherence of MPA, was caused by the dissociation of $\beta$-catenin from the cadherincatenin complex and the subsequent accumulation of $\beta$-catenin in the cytoplasm. This caused abnormal activation of the WNT/ $\beta$-catenin pathway. We also found that Wnt-1-specific overexpression and Axin 1 inhibition in MPA could explain the redistribution and cytoplasmic retention of $\beta$-catenin. Collectively, these findings suggest that an abnormality in the WNT/ $\beta$-catenin pathway could enhance the invasiveness of MPA through the overexpression of Wnt-1 and downregulation of Axin1 molecules.

Conclusions: Our data support the need for further research regarding the $\mathrm{WNT} / \beta$-catenin pathway and the need to develop novel targeted therapies for restoration of tumor cell adherence and improvement of the prognosis of MPA.
\end{abstract}

Keywords: Micropapillary-predominant adenocarcinoma (MPA); WNT/ $\beta$-catenin; Axin1; lymph node invasion

Submitted Mar 27, 2020. Accepted for publication Aug 13, 2020.

doi: $10.21037 /$ jtd-20-1495

View this article at: http://dx.doi.org/10.21037/jtd-20-1495

\section{Introduction}

Morbidity and mortality from non-small cell lung cancer (NSCLC), which constitutes approximately $80 \%$ of all lung cancers, have been increasing steadily since the 1930 s
$(1,2)$. In recent years, morbidity from lung adenocarcinoma has continued to increase in the Chinese population. Lung adenocarcinoma now become the most prevalence one within the NSCLC (3). Most fatal event of lung adenocarcinoma remains to be the terminal metastasis in 
the late stage of the disease (4).

Micropapillary-predominant adenocarcinoma (MPA) was first described by Silver and Askin in 1997 (5). Notable aggressive biological behaviors, including lymph node involvement and terminal metastasis, are common in this particular subtype. MPA is a poorly differentiated subtype of lung adenocarcinomas. Patients who have MPA, or only have micropapillary components in their lesions, will experience poorer clinical outcomes (6). Histological identification of MPA depending on its unique morphology under the microscopy, which has been defined by the International Association for the Study of Lung Cancer, the American Thoracic Society, and the European Respiratory Society (7).

Activation of the WNT signaling cascade is a critical molecular event in the embryonic development of mammals, it plays a role in hemostatic processes in tissues and stem cell regulation $(8,9)$. Thus, this molecular mechanism can accelerate tissue development and regeneration. However, it can also be utilized by cancer cells to promote uncontrollable cancer growth within the host bodies and lead to development of malignant lesions, such as leukemia, liver cancer, endometrial cancer, osteosarcoma, and lung cancer (10-13).

The WNT/ $\beta$-catenin pathway is regarded as the canonical WNT signaling pathway. It is triggered when Wnt-family proteins binding to specific cell membrane receptors, like Frizzled family of seven-transmembrane domain receptors and the low-density lipoprotein-receptorrelated proteins (14). Combination of these molecules can inhibit the axis inhibition protein 1 (Axin1) complex, which is competent in $\beta$-catenin degradation, resulting in cytosol accumulation and nuclear translocation of $\beta$-catenin. Nuclear $\beta$-catenin can heterodimerization with transcription factors of the lymphoid enhancer-binding factor/T cell factor family and upregulate specific oncogenes (e.g., Cyclin-D1 and c-Myc) (15). Recent studies demonstrated that enhanced $\mathrm{WNT} / \beta$-catenin signaling activity in breast, liver, and colorectal cancers were positively correlated with epithelial-to-mesenchymal transition (EMT) status and terminal metastasis (16-18).

The $\beta$-catenin destruction complex, as described above, plays a key role in determining the fate of $\beta$-catenin. This complex is composed of the scaffold protein Axin 1 and adenomatous polyposis coli (APC) together with some kinases, including glycogen synthase kinase $3 \beta$ (GSK-3 $\beta$ ) and casein kinase $1 \alpha(\mathrm{CK}-1 \alpha)(19)$. In the absence of Wnt ligand protein, cytoplasmic $\beta$-catenin can be phosphorylated by this complex, then undergo degradation (20). When Wnt/ $\beta$-catenin pathway is activated, the receptors are phosphorylated and inactivate the $\beta$-catenin destruction complex, thereby aiding in the accumulation and nuclear translocation of $\beta$-catenin (19).

In our study, we found that in MPA, upregulated Wnt1 and downregulation of Axin1 can over-activate WNT/ $\beta$-catenin signaling pathway and enhance the expression of downstream oncogene cyclinD1. The cadherin-catenin complex was also disrupted. These findings may provide us with a novel perspective on the molecular mechanisms associated with the aggressive biological behavior and poor clinical outcomes of MPA. The WNT/ $\beta$-catenin pathway may serve as a molecular target in improved treatments for MPA of the lung. We present the following article in accordance with the MDAR reporting checklist (available at http://dx.doi.org/10.21037/jtd-20-1495).

\section{Methods}

\section{Patients and specimens}

Twenty cases of lung adenocarcinoma tissue blocks for each MPA group and lepidic group were collected from the Department of Pathology at the Cancer Hospital of the University of Chinese Academy of Sciences between 2015 and 2018, the clinicopathological features of the patients were presented in Table 1. The study was conducted in accordance with the Declaration of Helsinki (as revised in 2013). The study was approved by the ethics committee of Zhejiang Cancer Hospital (No. IRB-2016-90). Written informed consent was provided by the patients from whom the tissue blocks were taken. For each case, hematoxylin and eosin-stained slides were reviewed under a microscope to confirm histological type. A case was classified as micropapillary or lepidic pattern predominant if the indicated component comprised more than $50 \%$ of the total neoplastic area.

\section{Reagents and antibodies}

Monoclonal primary antibodies against human $\alpha$-catenin (Cat\#2028-1), $\beta$-catenin (Cat\#1247-1), and E-cadherin (Cat\#1702-1) were purchased from Epitomics (Burlingame, CA, USA). Anti-Axin1 (Cat\#ab115205), anti-Wnt-1 (Cat\#ab15251), anti-Wnt-6 (Cat\#ab150588), anti-Wnt10a (Cat\#ab106522) and anti-Cyclin-D1 (Cat\#ab16663) antibodies were purchased from Abcam (Cambridge, MA, 
Table 1 Clinicopathological features of the patients

\begin{tabular}{|c|c|}
\hline Group & Num. \\
\hline \multicolumn{2}{|l|}{ MPA group } \\
\hline \multicolumn{2}{|l|}{ Gender } \\
\hline Male & 11 \\
\hline Female & 9 \\
\hline \multicolumn{2}{|l|}{ Age (years) } \\
\hline Male & 58 [33-76] \\
\hline Female & 52 [25-79] \\
\hline Differentiation & Poor \\
\hline \multicolumn{2}{|l|}{ T staging* } \\
\hline $\mathrm{T} 1$ & 10 \\
\hline T2 & 7 \\
\hline Т3 & 3 \\
\hline \multicolumn{2}{|l|}{ N staging } \\
\hline No & 9 \\
\hline N1 & 5 \\
\hline N2 & 6 \\
\hline \multicolumn{2}{|l|}{ M staging } \\
\hline Mo & 19 \\
\hline M1 & 1 \\
\hline \multicolumn{2}{|l|}{ Lepidic group } \\
\hline \multicolumn{2}{|l|}{ Gender } \\
\hline Male & 7 \\
\hline Female & 13 \\
\hline \multicolumn{2}{|l|}{ Age (years) } \\
\hline Male & 61 [36-72] \\
\hline Female & 55 [31-69] \\
\hline Differentiation & Well \\
\hline \multicolumn{2}{|l|}{ T staging } \\
\hline $\mathrm{T} 1$ & 18 \\
\hline $\mathrm{T} 2$ & 2 \\
\hline T3 & 0 \\
\hline \multicolumn{2}{|l|}{$\mathrm{N}$ staging } \\
\hline No & 20 \\
\hline N1 & 0 \\
\hline N2 & 0 \\
\hline \multicolumn{2}{|l|}{ M staging } \\
\hline MO & 20 \\
\hline M1 & 0 \\
\hline
\end{tabular}

*, the TNM stage were classified according to the WHO Classification of Tumors of the Lung, Pleura, Thymus and Heart ( $4^{\text {th }}$ edition, 2015).
USA). The fluorescent conjugated secondary antibody Goat Anti-Rabbit IgG H\&L (Alexa Fluor ${ }^{\circledR} 488$ ) was also obtained from Abcam (ab150081).

\section{Immunobistochemistry staining}

Tissue blocks were collected for each case. Paraffin sections ( $4 \mu \mathrm{m}$ thick) of either tissue or cell blocks were deparaffinized and rehydrated in xylene and a graded alcohol series. Endogenous peroxidase activity was blocked by incubation in $0.3 \%$ hydrogen peroxide, and the sections were blocked for 20 minutes with $10 \%$ goat serum. The sections were then incubated with primary antibodies including Wnt1 (1:100), Wnt6 (1:200), Wnt10a (1:400), Axin1 (1:100), E-cadherin (1:100), $\alpha$-catenin (1:100), $\beta$-catenin (1:200), and cyclinD1 (1:400) for 90 minutes at room temperature. After slides had been washed in phosphate-buffered saline, the slides were developed using 3,3'-diaminobenzidine (Dako Cytomation, Carpinteria, CA, USA), in accordance with the manufacturer's instructions, and counterstained with hematoxylin. Negative control slides were incubated with phosphate-buffered saline, instead of the primary antibody.

\section{Immunobistochemical staining evaluation}

All immunohistochemically stained slides were observed under the microscope. For each slide, hot spots of positive staining were identified at $100 \times$ power. Tumor positive scores (TPS) were calculated using 10 high-power phase of view fields (400x) within the hot spots, using the equation TPS $=$ (number of positive tumor cells/number of total tumor cells) $\times 100 \%$. Positive expression of a protein was defined as reliable and complete membrane or nuclear staining at any intensity when viewed under a magnification of $400 x$.

\section{Statistical analyses}

Comparisons of TPS for the MPA and lepidic groups were performed using the two-tailed unpaired Student's $t$-test. All statistical analyses were performed using GraphPad Prism ver.8.0 (GraphPad Software, La Jolla, CA, USA).

\section{Results}

\section{The cadherin-catenin complex was disrupted in MPA}

The rate of metastasis and lymph node invasion in MPA 
A
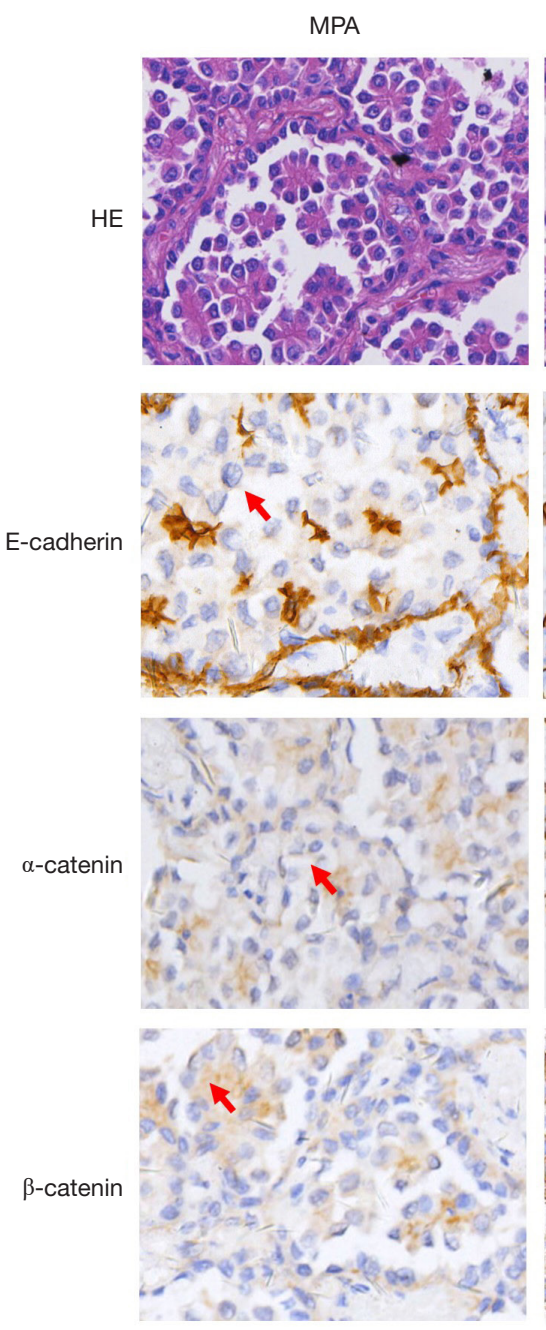

lepidic
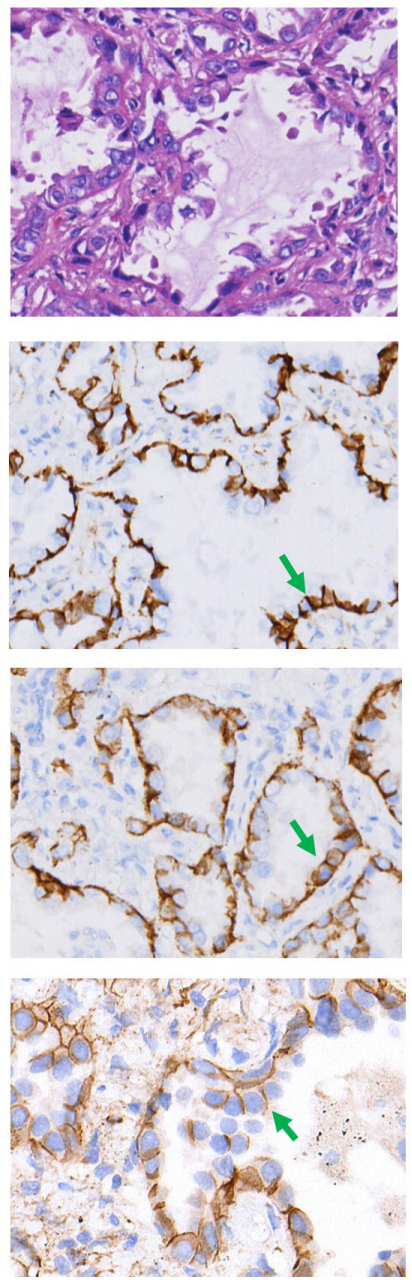

B
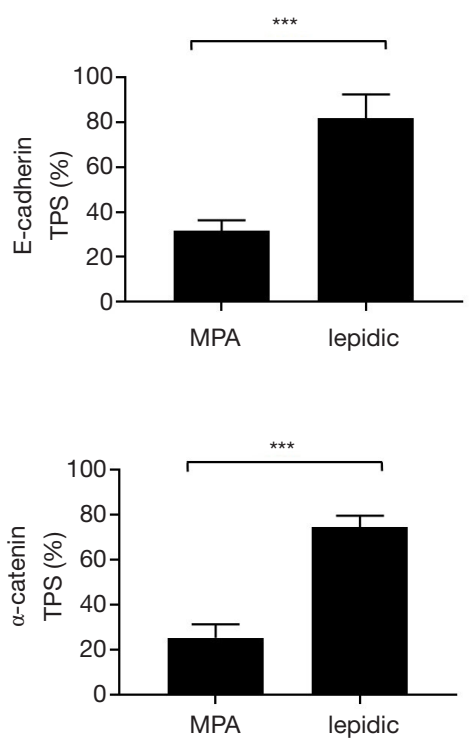

Figure 1 Disruption of cadherin-catenin complex in MPA of lung. (A) Cancer tissues of the MPA and lepidic groups were applied with $\mathrm{H} \& \mathrm{E}$ staining and IHC staining to show expression or the intracellular distribution of the three key molecules which compose the cadherincatenin complex (E-cadherin, $\alpha$-catenin and $\beta$-catenin). The pictures were taken under $400 \times$ power phase microscopically. Red arrows indicated typical negative tumor cells in the MPA group and the green ones indicated typical positively stained tumor cells in the lepidic group. (B) For each slide from both groups, the TPS of E-cadherin and $\alpha$-catenin were calculated by counting the positive rate of tumor cells in each 10 views at 400× power phases under microscope. Any convincing membrane staining of the cancer cells were considered to be positive. The data were then analyzed by application of two-tailed unpaired student's $t$-tests. ${ }^{* * *}$, indicated significant difference $(\mathrm{P}<0.0001)$. H\&E, hematoxylin and eosin; IHC, immunohistochemistry; MPA, Micropapillary-predominant adenocarcinoma; TPS, tumor positive score.

suggested reduced intercellular adhesion, which has been described by other researchers $(11,21)$. This characteristic of MPA prompting us to evaluate the intact of the cadherincatenin complex of the cancer cells. Immunohistochemical staining with anti-E-cadherin (TPS: $31.6 \%$ vs. $81.9 \%$, $\mathrm{P}<0.0001$ ) and anti- $\alpha$-catenin (TPS: $25.3 \%$ vs. $74.7 \%$, $\mathrm{P}<0.0001)$ antibodies indicated diminished protein expression, in terms of both intensity and percentage, in the cell membrane of MPA cases, compared to lepidic cases (Figures $1 A, B, S 1, S 2$ ). Concurrently, $\beta$-catenin was dissociated from the cell membrane in the MPA group (Figure 1A). These data suggested that destruction of the cadherin-catenin complex may have contributed to the poor intercellular adherence of MPA. 

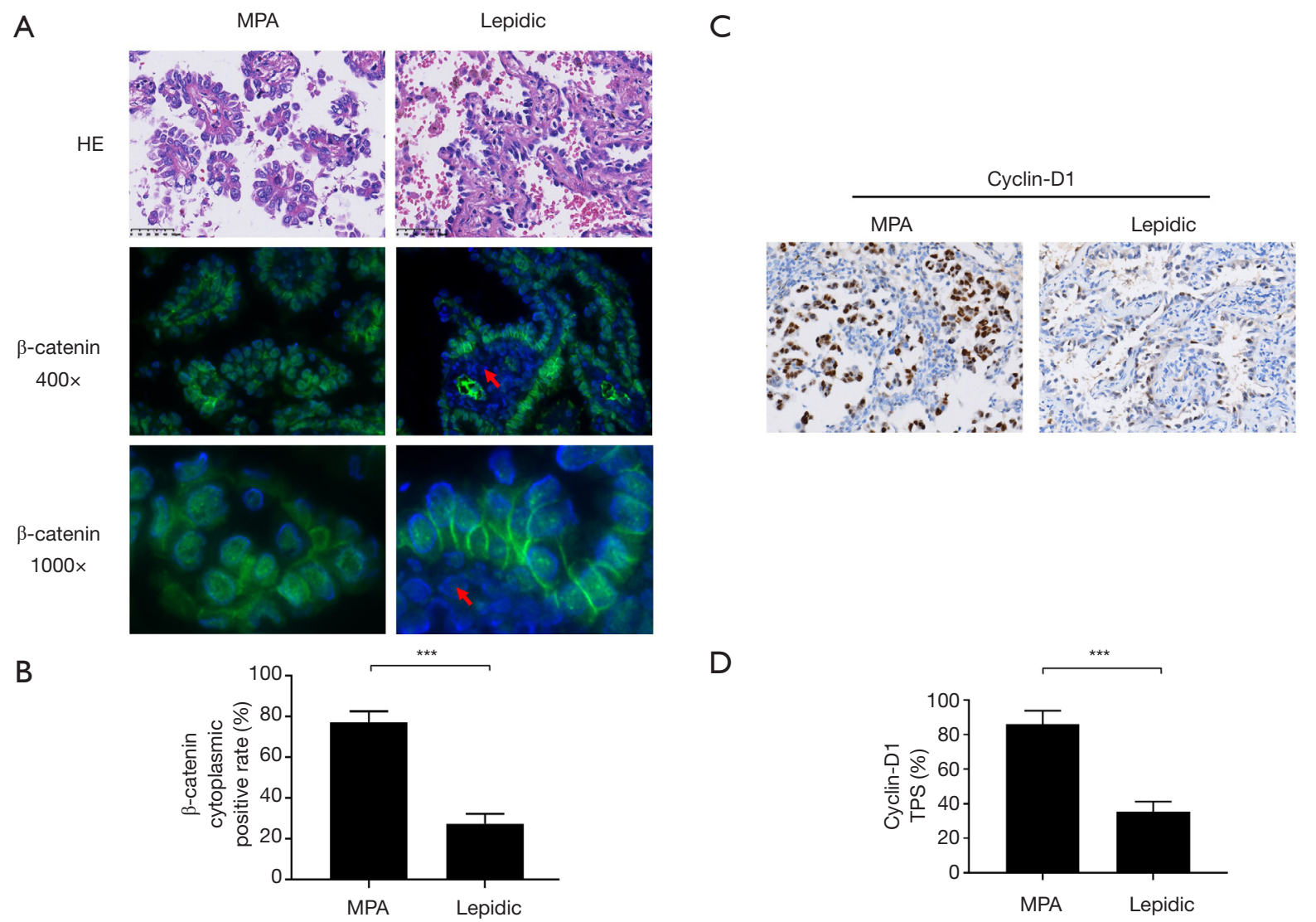

Figure 2 Cytoplasmic accumulation of $\beta$-catenin enhanced the WNT/ $\beta$-catenin signaling activity in the MPA of lung. (A) Cancer tissues of the MPA and lepidic groups were applied with H\&E staining and IHC staining with fluorescent conjugated secondary antibody to show the intracellular distribution of $\beta$-catenin. The pictures were taken under microscope at $400 \times$ and $1,000 \times$ (oil lens) power phase. The red arrows indicated interstitial cells. (B) Cyclin-D1 expression in both groups were evaluated by IHC staining to show the activity of WNT/ $\beta$-catenin signaling activity in cancer cells. The pictures were taken under microscope at 400× power phase. (C,D) Within both of the groups, cytoplasm positive rates of $\beta$-catenin were calculated by counting tumor cells with dramatic cytoplasmic but no clear membrane fluorescent signal (C) and Cyclin-D1 TPS were calculated by counting tumor cells with convincing nuclear staining (D). For each slide, 10 views at $400 \times$ power phases were analyzed. The data were then analyzed by application of two-tailed unpaired student's $t$-tests. Three asterisks indicated significant difference $(\mathrm{P}<0.0001)$. H\&E, hematoxylin and eosin; IHC, immunohistochemistry; MPA, Micropapillary-predominant adenocarcinoma; TPS, tumor positive score.

\section{Cytoplasmic accumulation of $\beta$-catenin induced canonical Wnt signaling and may bave contributed to cadberin- catenin complex disruption in MPA}

We then sought to identify the mechanism by which cadherin-catenin complex disruption was initiated in MPA. It has been reported that activation of the $W \mathrm{nt} / \beta$-catenin pathway may favor the degradation of E-cadherin (22). Based on this finding, together with the dissociation of $\beta$-catenin from the cell membrane shown in Figure $1 \mathrm{~A}$, we proceeded to perform immunofluorescent staining of $\beta$-catenin to allow observation of the intracellular distribution of $\beta$-catenin at high resolution. The results were presented in Figure 2A,B. Accumulated cytoplasmic $\beta$-catenin was more common in MPA than in the lepidic subtype $(77.2 \%$ vs. $27.3 \%, \mathrm{P}<0.001)$. To verify downstream $W n t / \beta$-catenin signaling activity, the expression of Cyclin-D1, a key product of this pathway, was evaluated in typical components from both groups. As shown in Figure $2 C$, a higher expression rate of Cyclin-D1 was observed in the MPA group $(87.9 \%$ vs. $35.5 \%, \mathrm{P}<0.001$, Figure $2 D$ ), indicating more active $W n t / \beta$-catenin signaling 
activity. In conclusion, cytoplasmic accumulation of $\beta$-catenin disrupted the cell membrane cadherin-catenin complex, leading to low intercellular adherence. In contrast, it enhanced $\mathrm{Wnt} / \beta$-catenin signaling and increased the production of relevant oncogenes in MPA.

\section{Wnt-1 expression was specifically increased in MPA}

Abnormal $W n t / \beta$-catenin signaling resulting from the overexpression of various Wnt ligands has been reported in NSCLC. However, details of the mechanism by which excessive $W n t / \beta$-catenin signaling is triggered in MPA have been unclear. We analyzed the expression levels of three canonical Wnt pathway ligands-Wnt-1, Wnt-6, and Wnt-10a-in cancer cells. The results indicated a moderate increase in the percentage of cells with Wnt-1 expression $(90.6 \%$ vs. $71.4 \%, \mathrm{P}<0.05)$ and a dramatically enhanced staining intensity (Figure 3 ) in the MPA group. In contrast, Wnt-6 $(82.3 \%$ vs. $85.6, \mathrm{P}=0.134)$ and Wnt10a $(18.7 \%$ vs. $22.4 \%, \mathrm{P}=0.11)$ were present at similar levels in the two groups (Figure 3), indicating that Wnt/ $\beta$-catenin signaling in MPA was triggered by a specific type of Wnt molecule.

\section{Axin1 expression was downregulated in MPA, especially in cases with lymph node invasion}

Axin1 protein is a scaffold protein in the cytoplasmic $\beta$-catenin-destruction complex. Axin 1 deficiency has been shown to cause overactive $\mathrm{Wnt} / \beta$-catenin signaling in various malignant neoplasms. We explored Axin 1 expression status in the MPA and lepidic groups. After immunohistochemical staining had been performed, slides from the two groups were evaluated for staining intensity and TPS of Axin 1 in the histologically typical components. The results, shown in Figure $4 A$, indicated that almost all tumor cells in the lepidic group were strongly positive; in the MPA group, the majority of tumor components were only weakly to moderately positive and the percentage with positive expression was smaller than the percentage in the lepidic group (63.5\% vs. $95.0 \%, \mathrm{P}<0.001)$. These findings demonstrate that MPA expressed less Axin 1 than the lepidic subtype of lung adenocarcinoma. We also evaluated the TPS of Axin 1 in both lymph node invasion-negative and lymph node invasion-positive MPA cases to explore whether Axin1 expression was essential for lymph node invasion. The results are presented in Figure 4B. Axin 1 was differentially expressed in the lymph node invasion- positive and lymph node invasion-negative groups (61.46\% vs. $68.04 \%, \mathrm{P}=0.018$ ). Taken together, these results indicate that diminished Axin1 expression may affect the invasiveness of lung adenocarcinoma, in addition to its histological differentiation.

\section{Discussion}

According to the histologic classification of the International Association for the Study of Lung Cancer, American Thoracic Society, and European Respiratory Society, lung adenocarcinoma has five predominant subtypes: lepidic, acinar, papillary, micropapillary, and solid $(7,23)$. The micropapillary subtype behaves more aggressively and has poorer clinical outcomes than the other subtypes. Pathologically, MPA is defined with a tissue structure that is organized in delicate papillary tufts and lacks fibrovascular cores (24). Clinically, it induces higher metastatic rate and more rapid tumor proliferation $(25,26)$. However, because MPA was only recently identified, its biological behavior has not been explained. Our study attempts to explore the mechanism by which MPA derived more invasiveness and we find abnormal Wnt-1 and Axin 1 expression in MPA, which may interpret as the reason for elevated activity of the WNT/ $\beta$-catenin pathway. These molecular events may promote lymph nodes invasion of MPA in our cases.

In some type of cancers, activation of WNT signaling can coupled with dissociation of $\beta$-catenin from the cell membrane and break down the cadherin-catenin complex, which is essential for maintenance of intercellular adherence (9). Our data presented downregulation of E-cadherin and $\alpha$-catenin on the cell membrane (Figure 1). This may explain the low intercellular adhesion of MPA, which frequently leads to intra-alveolar tumor spread (27). However, the role of WNT pathway ligands in NSCLC is presumably complicated. Some ligands, such as Wnt-1, Wnt-2, and Wnt-3, can activate the canonical WNT pathway and directly promote cancer growth (23-26). Others, including Wnt-5a and Wnt-11, serve as non-canonical WNT pathway activators. They can block canonical WNT signaling as well as inducing other malignant behavior (such as EMT) by $\beta$-catenin-independent mechanisms $(28,29)$. These findings led us to hypothesize that an expression panel of Wnt family members would interact with each other in cancer progression. Our data were partially consisted with this interpretation and we showed that Wnt-1 expression was enhanced in MPA (Figure 3). However, the expression levels of other Wnt members, such as Wnt-6 and Wnt- 
A

HE
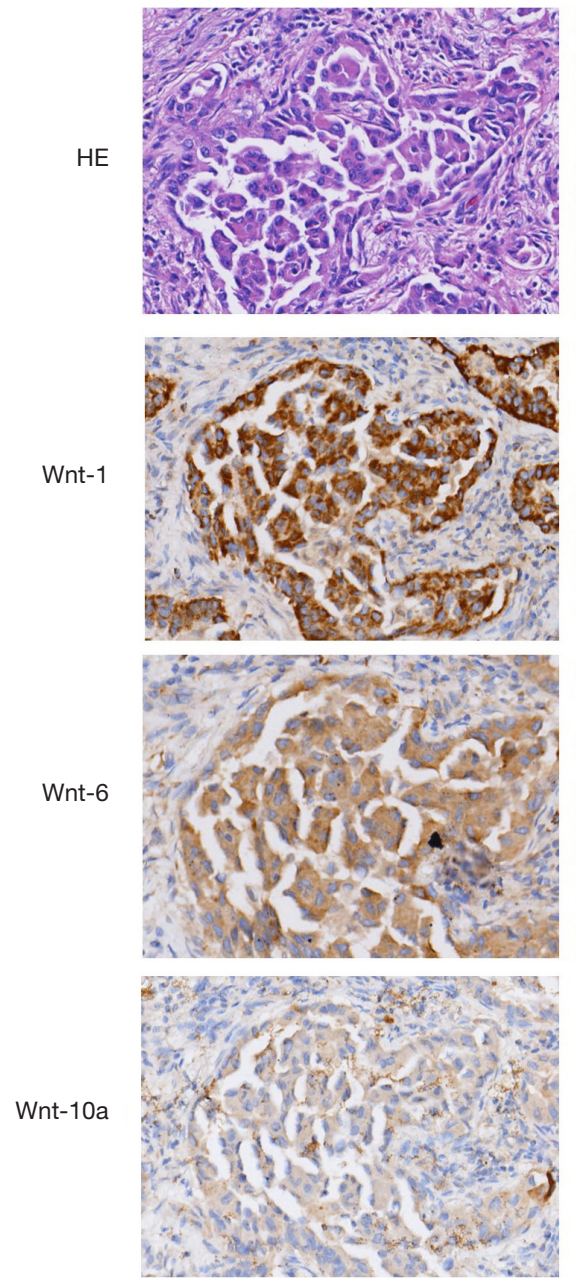
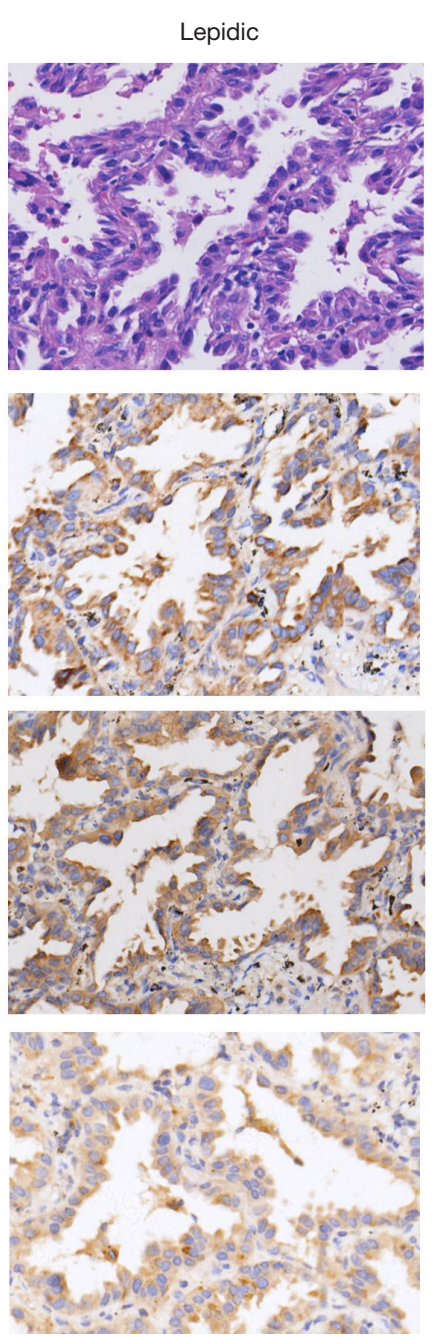

B
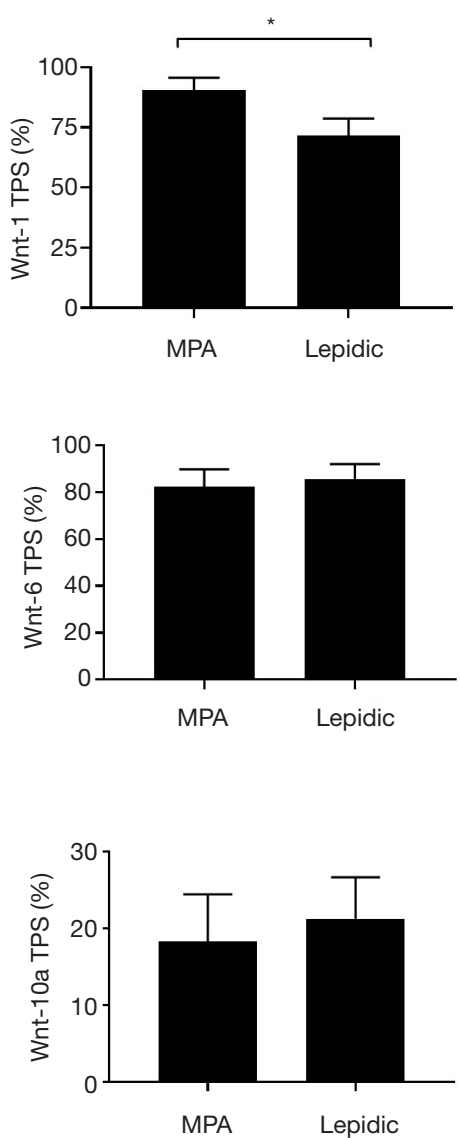

Figure 3 Dramatic and specific Wnt-1 over-expression in the MPA of lung. (A) Cancer tissues of the MPA and lepidic groups were applied with H\&E staining and IHC staining to show the expression of Wnt-1, Wnt-6 and Wnt-10a in cancer cells. The pictures were taken under microscope at 400x power phase. (B) For each slide from both groups, the TPS of Wnt-1, Wnt-6 and Wnt-10a were calculated by counting the positive rate of tumor cells in each 10 views at $400 \times$ power phases under microscope. Any convincing cytoplasmic staining of the cancer cells were considered to be positive. The data were then analyzed by application of two-tailed unpaired student's $t$-tests. *, indicated statistical difference $(\mathrm{P}<0.05)$. H\&E, hematoxylin and eosin; IHC, immunohistochemistry; MPA, Micropapillary-predominant adenocarcinoma; TPS, tumor positive score.

10a, did not show significant difference between the MPA and lepidic groups. These results suggest the existence of complicated and dynamic Wnt-regulation networks in different pathologic patterns of lung adenocarcinoma.

$\beta$-catenin expression has been identified in up to $51 \%$ of lung adenocarcinomas (30). Jin et al. reported that the expression of $\beta$-catenin in the cell membrane was associated with a higher survival rate [hazard ratio (HR): 0.53 ; 95\% confidence interval (CI): 0.32-0.87].
In contrast, $\beta$-catenin expression patterns in the cytoplasm and nucleus were associated with a poor survival rate, with HRs of 1.63 (95\% CI: 1.34-1.99) and 3.15 (95\% CI: $1.97-5.05)$, respectively (31). These data demonstrated that excessive intracellular $\beta$-catenin may help cancer cells become more invasive. Nuclear $\beta$-catenin accumulation was also correlated with epidermal growth factor receptor (EGFR) mutations and resistance to gefitinib $(32,33)$, which cause these cancers 
A

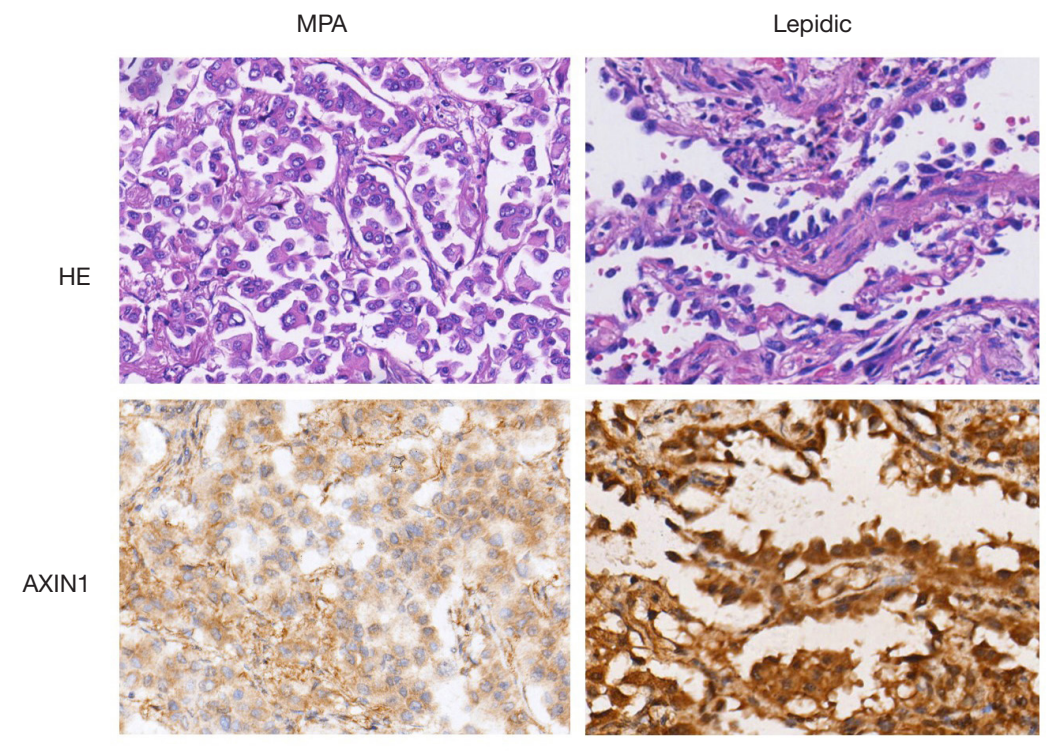

B

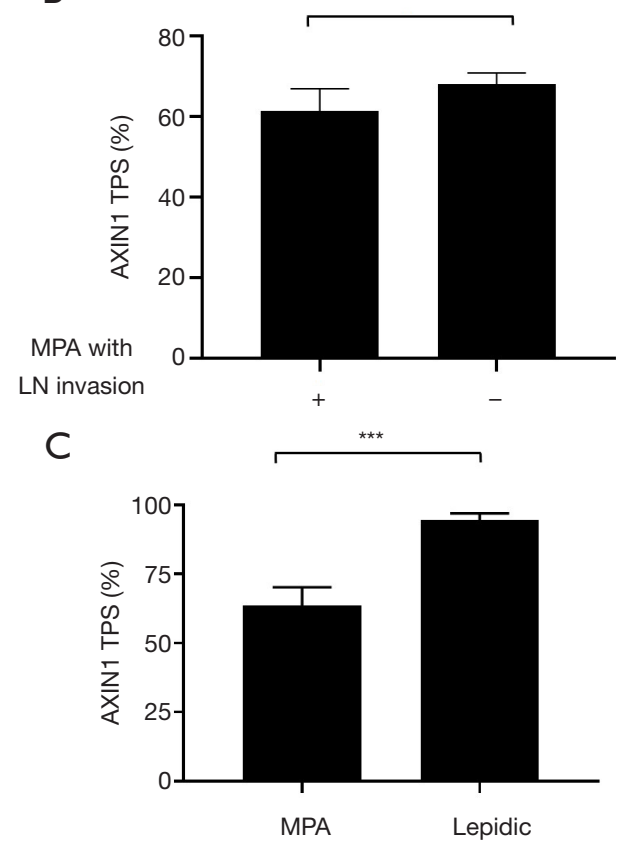

Figure 4 Expression of WNT/ $\beta$-catenin signaling negative regulator Axin1 was decreased in MPA of lung, especially in cases with lymph node invasion. (A) Cancer tissues of the MPA and lepidic groups were applied with H\&E staining and IHC staining to show the intension of cytoplasmic Axin1. The pictures were taken under microscope at 400× power phase. (B) For each slide from both groups, the TPS of Axin 1 was calculated by counting the positive rate of tumor cells in each 10 views at $400 \times$ power phases under microscope. Any convincing cytoplasmic staining of the cancer cells were considered to be positive. The data were then analyzed by application of two-tailed unpaired student's $t$-tests. *, indicated statistical difference $(\mathrm{P}<0.05)$; ${ }^{* * *}$, indicated significant difference $(\mathrm{P}<0.001)$. (C) Within the MPA group, MPA expression comparison between cases with or without lymph node invasion by the same method indicated lower TPS in the ones with lymph node invasion.

to be insensitive to treatment with EGFR-tyrosine kinase inhibitor. However, the difference in $\beta$-catenin distribution between well-differentiated and poorly differentiated lung adenocarcinomas remains unknown. Our study firstly discovers that poorly differentiated lung adenocarcinomas, such as MPA, involve more extensive intracellular $\beta$-catenin translocation, compared to well-differentiated lung adenocarcinomas (Figure $2 A, C$ ). Our data provide a possible explanation for highly aggressive phenotype of this particular histological subtype of lung adenocarcinoma.

The Axin 1 protein acts as a scaffold in a destruction complex, whereby $\beta$-catenin is phosphorylated by glycogen synthase kinase $3 \beta$ and undergoes ubiquitination-dependent degradation. Disruption of this complex will lead to excessive accumulation of $\beta$-catenin and downstream oncogene transcription. Axin1 protein dysfunction caused by gene mutations has been reported in various tumors, including hepatocellular, colorectal, prostate, and gastric carcinomas (34-37). In our study, we found that Axin 1 expression was lower in the MPA subtype than in the welldifferentiated lepidic subtype in terms of both percentage and staining intensity (Figure 4A,B). Evidence for a similar relationship between a silent Axin1 mutation and prognosis has been demonstrated in esophageal cancer (38), whereas the role of Axin1 in the biological behavior of lung cancers remains a mystery. Our results suggested that reduced Axin1 expression and a dysfunctional $\beta$-catenin destruction complex serve as a potential mechanism for cytoplasmic accumulation of $\beta$-catenin and activation of downstream signal transduction in MPA, which can further facilitate lymph nodes invasion of the MPA.

\section{Conclusions}

In summary, our results indicate that over activation of the WNT/ $\beta$-catenin signaling pathway leads to disruption of the cadherin-catenin complex and causes decreased intercellular adherence in MPA of the lung. Elevated 
expression of Wnt-1 and downregulation of Axin1 may play key roles in activation of this pathway. Our investigation may provide the potential target for development of medical strategies focused on the $\mathrm{WNT} / \beta$-catenin pathway to improving the prognosis of MPA of the lung in the future.

\section{Acknowledgments}

Funding: This work was supported by grants from the National Natural Science Foundation of China (No. 81702851), Zhejiang Province Medical and Health Science and Technology Project (No. 2018KY030) and Zhejiang Provincial Natural Science Foundation of China (LGF20H160008).

\section{Footnote}

Reporting Checklist: The authors have completed the MDAR reporting checklist. Available at http://dx.doi.org/10.21037/ jtd-20-1495

Data Sharing Statement: Available at http://dx.doi. org/10.21037/jtd-20-1495

Conflicts of Interest: All authors have completed the ICMJE uniform disclosure form (available at http://dx.doi. org/10.21037/jtd-20-1495). The authors have no conflicts of interest to declare.

Ethical Statement: The authors are accountable for all aspects of the work in ensuring that questions related to the accuracy or integrity of any part of the work are appropriately investigated and resolved. The study was conducted in accordance with the Declaration of Helsinki (as revised in 2013). The study was approved by the ethics committee of Zhejiang Cancer Hospital (No. IRB-201690). Written informed consent was provided by the patients from whom the tissue blocks were taken.

Open Access Statement: This is an Open Access article distributed in accordance with the Creative Commons Attribution-NonCommercial-NoDerivs 4.0 International License (CC BY-NC-ND 4.0), which permits the noncommercial replication and distribution of the article with the strict proviso that no changes or edits are made and the original work is properly cited (including links to both the formal publication through the relevant DOI and the license). See: https://creativecommons.org/licenses/by-nc-nd/4.0/.

\section{References}

1. Ridge CA, McErlean AM, Ginsberg MS. Epidemiology of lung cancer. Semin Intervent Radiol 2013;30:93-8.

2. Zheng R, Zeng H, Zuo T, et al. Lung cancer incidence and mortality in China, 2011. Thorac Cancer 2016;7:94-9.

3. Lu S, Yu Y, Yang Y. Retrospect and Prospect for Lung Cancer in China: Clinical Advances of Immune Checkpoint Inhibitors. Oncologist 2019;24:S21-S30.

4. Herbst RS, Heymach JV, Lippman SM. Lung cancer. N Engl J Med 2008;359:1367-80.

5. Silver SA, Askin FB. True papillary carcinoma of the lung: a distinct clinicopathologic entity. Am J Surg Pathol 1997;21:43-51.

6. Park JK, Kim JJ, Moon SW, et al. Lymph node involvement according to lung adenocarcinoma subtypes: lymph node involvement is influenced by lung adenocarcinoma subtypes. J Thorac Dis 2017;9:3903-10.

7. Travis WD, Brambilla E, Noguchi M, et al. International association for the study of lung cancer/american thoracic society/european respiratory society international multidisciplinary classification of lung adenocarcinoma. J Thorac Oncol 2011;6:244-85.

8. Hogan BL, Barkauskas CE, Chapman HA, et al. Repair and regeneration of the respiratory system: complexity, plasticity, and mechanisms of lung stem cell function. Cell Stem Cell 2014;15:123-38.

9. Clevers H, Loh KM, Nusse R. Stem cell signaling. An integral program for tissue renewal and regeneration: Wnt signaling and stem cell control. Science 2014;346:1248012.

10. Wang Y, Krivtsov AV, Sinha AU, et al. The Wnt/betacatenin pathway is required for the development of leukemia stem cells in AML. Science 2010;327:1650-3.

11. Cha MJ, Lee HY, Lee KS, et al. Micropapillary and solid subtypes of invasive lung adenocarcinoma: clinical predictors of histopathology and outcome. J Thorac Cardiovasc Surg 2014;147:921-8.e2.

12. Chan DW, Chan CY, Yam JW, et al. Prickle-1 negatively regulates $\mathrm{Wnt} /$ beta-catenin pathway by promoting Dishevelled ubiquitination/degradation in liver cancer. Gastroenterology 2006;131:1218-27.

13. Goad J, Ko YA, Kumar M, et al. Oestrogen fuels the growth of endometrial hyperplastic lesions initiated by overactive Wnt/beta-catenin signalling. Carcinogenesis 2018;39:1105-16.

14. Mikels AJ, Nusse R. Wnts as ligands: processing, secretion and reception. Oncogene 2006;25:7461-8.

15. MacDonald BT, Tamai K, He X. Wnt/beta-catenin 
signaling: components, mechanisms, and diseases. Dev Cell 2009;17:9-26.

16. Wu ZQ, Li XY, Hu CY, et al. Canonical Wnt signaling regulates Slug activity and links epithelial-mesenchymal transition with epigenetic Breast Cancer 1, Early Onset (BRCA1) repression. Proc Natl Acad Sci U S A 2012;109:16654-9.

17. Wang Y, Bu F, Royer C, et al. ASPP2 controls epithelial plasticity and inhibits metastasis through betacatenin-dependent regulation of ZEB1. Nat Cell Biol 2014;16:1092-104.

18. Tenbaum SP, Ordonez-Moran P, Puig I, et al. betacatenin confers resistance to $\mathrm{PI} 3 \mathrm{~K}$ and $\mathrm{AKT}$ inhibitors and subverts FOXO3a to promote metastasis in colon cancer. Nat Med 2012;18:892-901.

19. Metcalfe C, Mendoza-Topaz C, Mieszczanek J, et al. Stability elements in the LRP6 cytoplasmic tail confer efficient signalling upon DIX-dependent polymerization. J Cell Sci 2010;123:1588-99.

20. Latres E, Chiaur DS, Pagano M. The human F box protein beta-Trcp associates with the Cul1/Skp1 complex and regulates the stability of beta-catenin. Oncogene 1999; 18:849-54.

21. Miyoshi T, Shirakusa T, Ishikawa Y, et al. Possible mechanism of metastasis in lung adenocarcinomas with a micropapillary pattern. Pathol Int 2005;55:419-24.

22. Heuberger J, Birchmeier W. Interplay of cadherinmediated cell adhesion and canonical Wnt signaling. Cold Spring Harb Perspect Biol 2010;2:a002915.

23. Akiri G, Cherian MM, Vijayakumar S, et al. Wnt pathway aberrations including autocrine $\mathrm{Wnt}$ activation occur at high frequency in human non-small-cell lung carcinoma. Oncogene 2009;28:2163-72.

24. You L, He B, Xu Z, et al. Inhibition of Wnt-2-mediated signaling induces programmed cell death in non-small-cell lung cancer cells. Oncogene 2004;23:6170-4.

25. Huang CL, Liu D, Ishikawa S, et al. Wnt1 overexpression promotes tumour progression in non-small cell lung cancer. Eur J Cancer 2008;44:2680-8.

26. Nakashima N, Liu D, Huang CL, et al. Wnt3 gene expression promotes tumor progression in non-small cell lung cancer. Lung Cancer 2012;76:228-34.

27. Warth A, Muley T, Kossakowski CA, et al. Prognostic Impact of Intra-alveolar Tumor Spread in Pulmonary Adenocarcinoma. Am J Surg Pathol 2015;39:793-801.

28. Bartis D, Csongei V, Weich A, et al. Down-regulation of canonical and up-regulation of non-canonical Wnt signalling in the carcinogenic process of squamous cell lung carcinoma. PLoS One 2013;8:e57393.

29. Kikuchi A, Yamamoto H, Sato A, et al. Wnt5a: its signalling, functions and implication in diseases. Acta Physiol (Oxf) 2012;204:17-33.

30. Kren L, Hermanova M, Goncharuk VN, et al. Downregulation of plasma membrane expression/cytoplasmic accumulation of beta-catenin predicts shortened survival in non-small cell lung cancer. A clinicopathologic study of 100 cases. Cesk Patol 2003;39:17-20.

31. Jin J, Zhan P, Katoh M, et al. Prognostic significance of beta-catenin expression in patients with non-small cell lung cancer: a meta-analysis. Transl Lung Cancer Res 2017;6:97-108.

32. Suzuki M, Shigematsu H, Nakajima T, et al. Synchronous alterations of Wnt and epidermal growth factor receptor signaling pathways through aberrant methylation and mutation in non small cell lung cancer. Clin Cancer Res 2007;13:6087-92.

33. Fang $\mathrm{X}, \mathrm{Gu} \mathrm{P}, \mathrm{Zhou} \mathrm{C}$, et al. beta-Catenin overexpression is associated with gefitinib resistance in non-small cell lung cancer cells. Pulm Pharmacol Ther 2014;28:41-8.

34. Hale G, Liu X, Hu J, et al. Correlation of exon 3 betacatenin mutations with glutamine synthetase staining patterns in hepatocellular adenoma and hepatocellular carcinoma. Mod Pathol 2016;29:1370-80.

35. Picco G, Petti C, Centonze A, et al. Loss of AXIN1 drives acquired resistance to WNT pathway blockade in colorectal cancer cells carrying RSPO3 fusions. EMBO Mol Med 2017;9:293-303.

36. Yardy GW, Bicknell DC, Wilding JL, et al. Mutations in the AXIN1 gene in advanced prostate cancer. Eur Urol 2009;56:486-94.

37. Zhu B, Wang DN, Gong XM, et al. [Expression of AXIN and MACC1 in Gastric Carcinoma and Its Clinical Significance]. Sichuan Da Xue Xue Bao Yi Xue Ban 2018;49:59-64.

38. Nakajima M, Fukuchi M, Miyazaki T, et al. Reduced expression of Axin correlates with tumour progression of oesophageal squamous cell carcinoma. Br J Cancer 2003;88:1734-9.

Cite this article as: Zhu L, Yang S, Zheng L, Zhang G, Cheng G. WNT/ $\beta$-catenin pathway activation via $W n t 1$ overexpression and Axin 1 downregulation correlates with cadherin-catenin complex disruption and increased lymph node involvement in micropapillary-predominant lung adenocarcinoma. J Thorac Dis 2020;12(10):5906-5915. doi: 10.21037/jtd-20-1495 
$\mathrm{HE}$
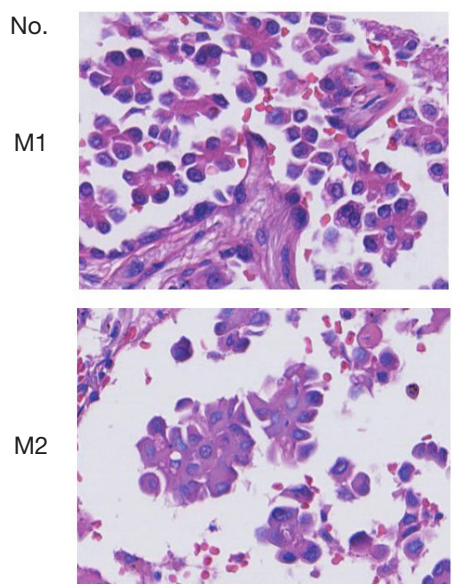

$\mathrm{M} 3$

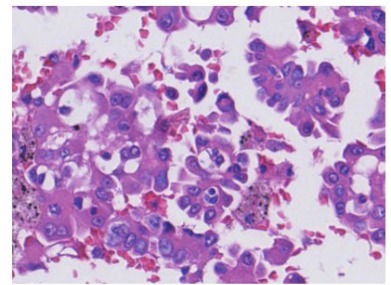

M5
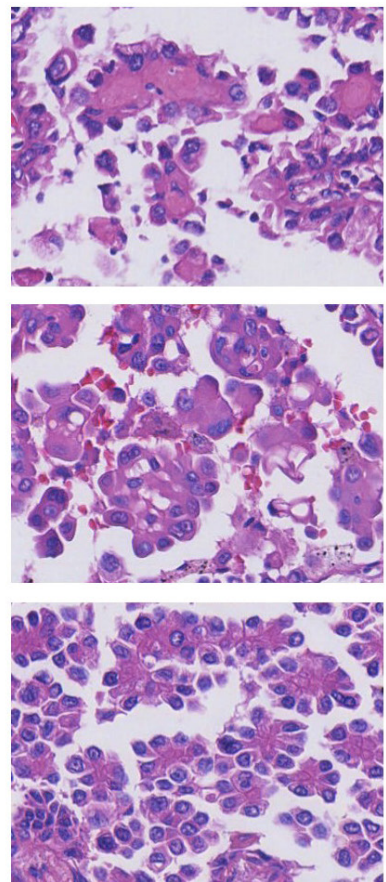

IHC: E-cadherin
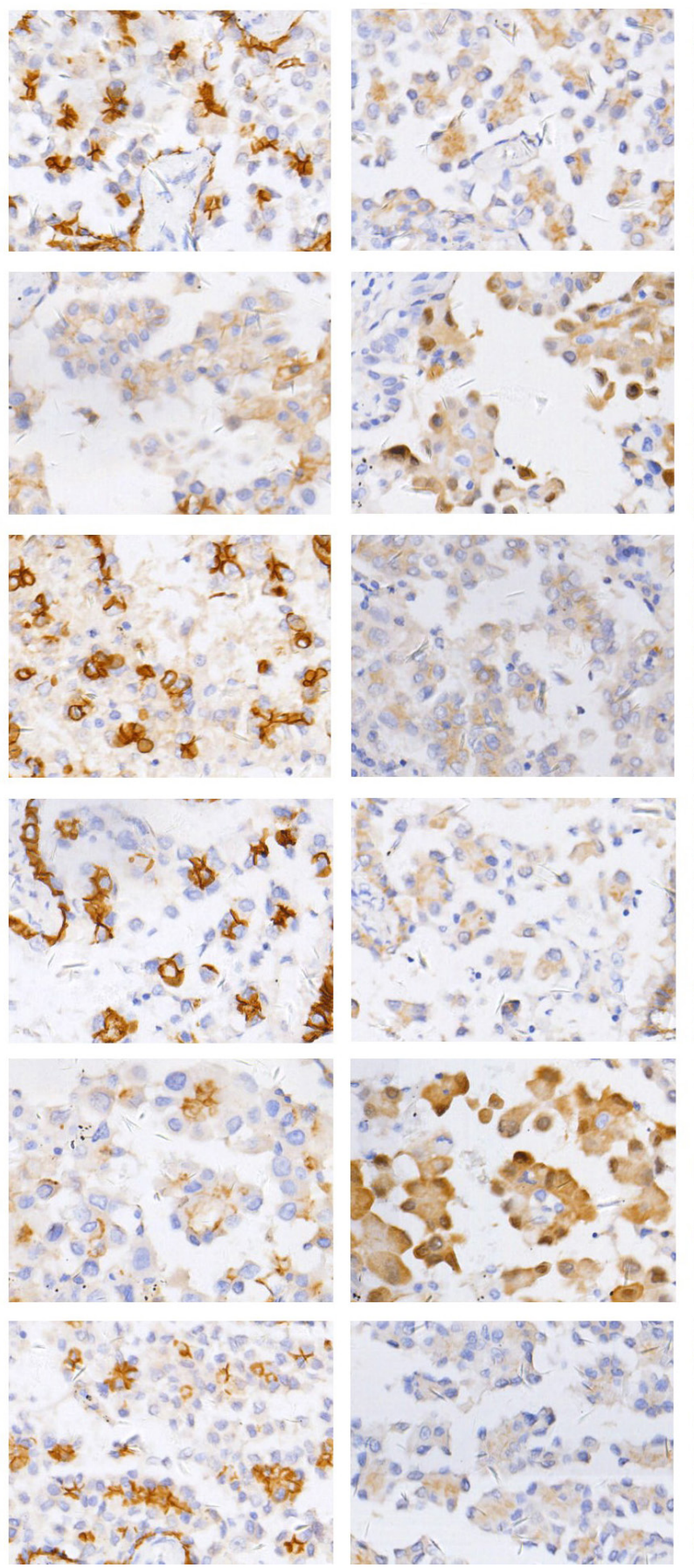
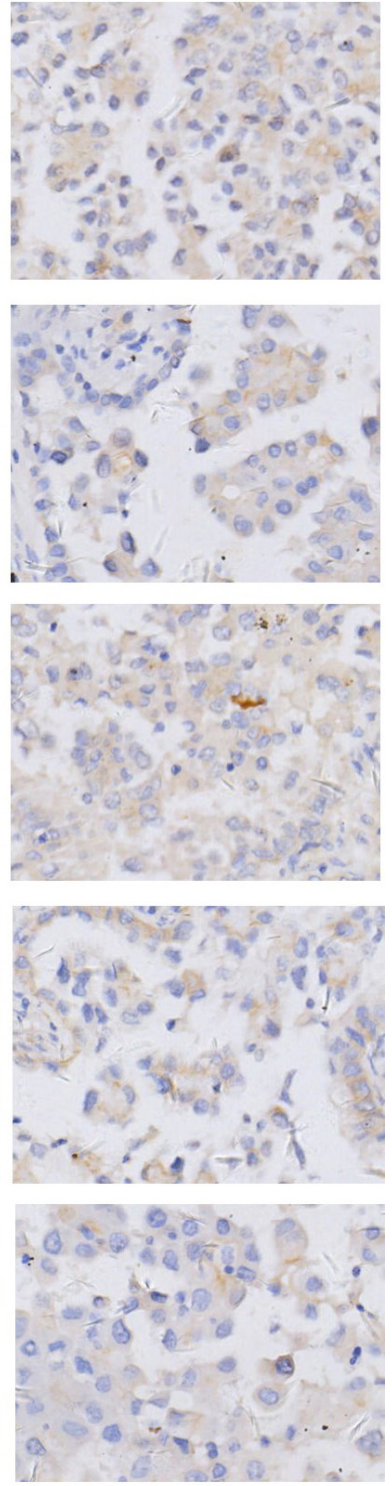

Figure S1 Expression of E-cadherin, b-catenin and a-catenin in the MPA. M1-6 stands for MPA case 1-6. All the pictures were taken under $40 \times$ power phase. 
HE

No.

L1

L2

L3
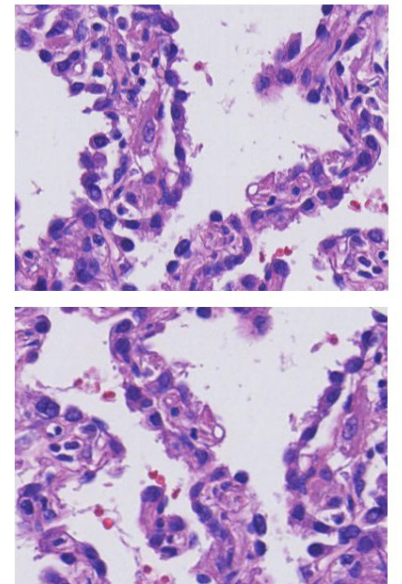
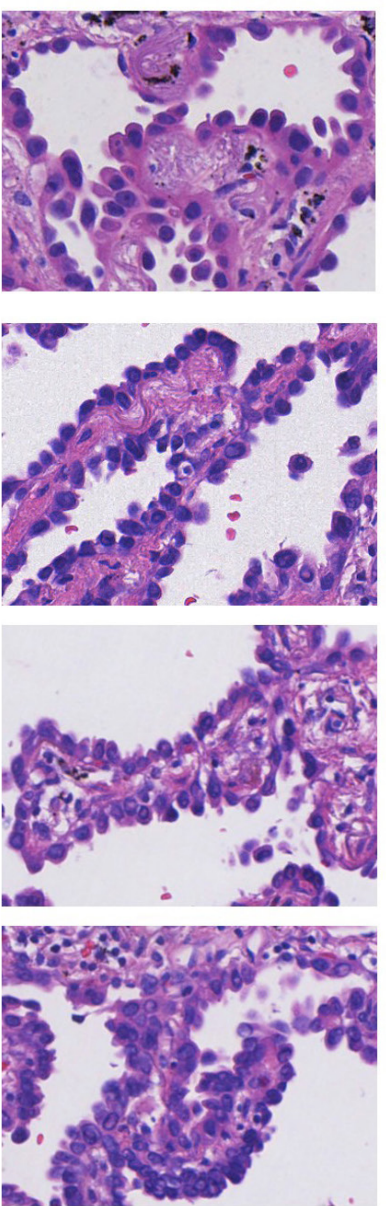

IHC: $\alpha$-catenin
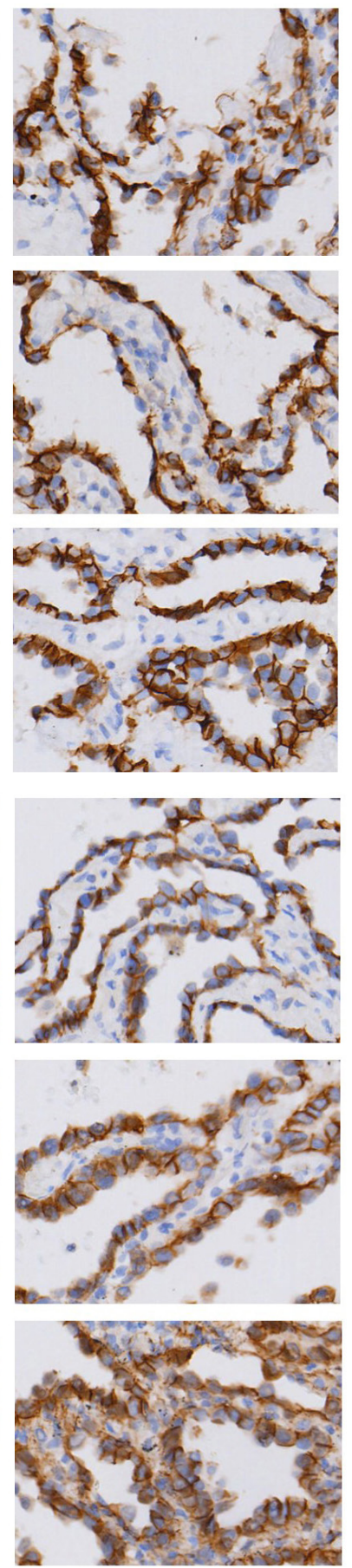

IHC: $\beta$-catenin
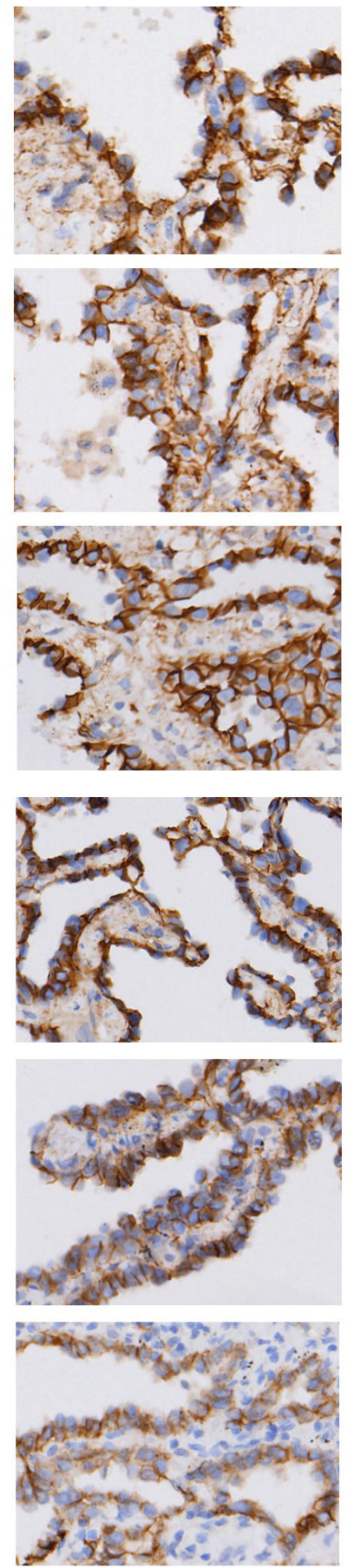

IHC: $\alpha$-catenin
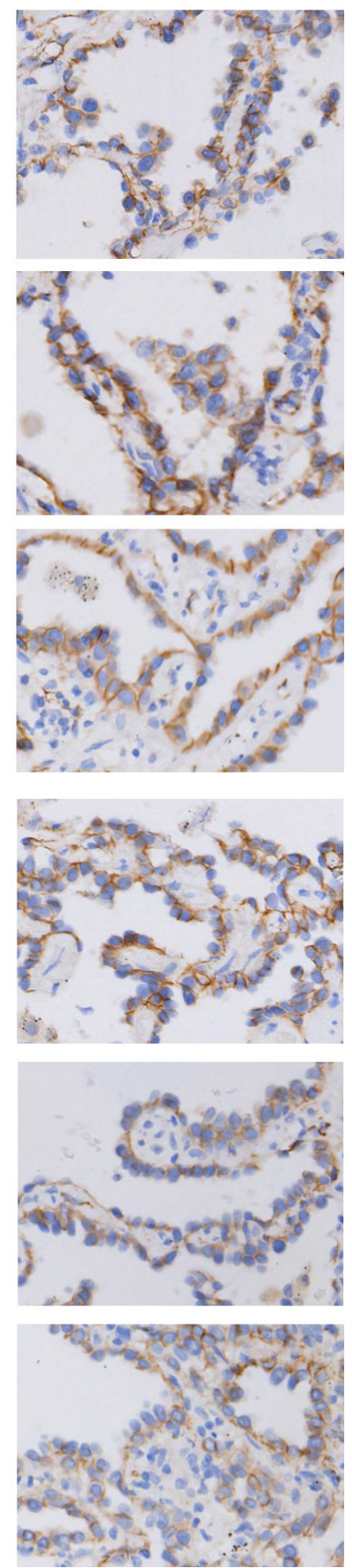

Figure S2 Expression of E-cadherin, b-catenin and a-catenin in the lepidic adenocarcinoma. L1-6 stands for lepidic case 1-6. All the pictures were taken under $40 \times$ power phase. 